Randomized Trial

\title{
Pulsed Radiofrequency of the Sacral Roots Improves the Success Rate of Superior Hypogastric Plexus Neurolysis in Controlling Pelvic and Perineal Cancer Pain. Randomized Trial
}

Diab Fuad Hetta, MD¹, Ashraf Amin Mohamed, MD¹, Rania Mohammed Abdel Emam, MD', Fatma Ahmed Abd El Aal, MD², and Marina Emeel Helal, MD'

From: ${ }^{1}$ South Egypt Cancer Institute, Assuit University, Assuit City, Egypt; ${ }^{2}$ Assuit University Hospital, Assuit University, Assuit City, Egypt

Address Correspondence: Diab Fuad Hetta, MD South Egypt Cancer Institute, Assuit University

71111, El-methaque St., city district, Assuit city, Egypt

E-mail: diabhetta25@ gmail.com

Disclaimer: There was no external funding in the preparation of this manuscript. Conflict of interest: Each author certifies that he or she, or a member of his or her immediate family, has no commercial association

(i.e., consultancies, stock ownership, equity interest, patent/licensing arrangements, etc.) that might pose a conflict of interest in connection

with the submitted manuscript.

Manuscript received: 06-01-2019

Revised manuscript received: 07-06-2019

Accepted for publication: 09-12-2019

Free full manuscript: www. painphysicianjournal.
Background: Superior hypogastric plexus neurolytic (SHP-N) block is the mainstay management for pelvic cancer pain of visceral origin when oral opioids fail due to inefficacy or intolerance to side effects. Unfortunately, SHP-N has the potential to control pelvic pain in $62 \%-72 \%$ of patients at best, because chronic pelvic pain may assume additional characteristics other than visceral.

Objective: Combining SHP-N with pulsed radiofrequency (PRF) of the sacral roots might block most of the pain characteristics emanating from the pelvic structures and improve the success rate of SHP-N in controlling pelvic and perineal cancer pain.

Study Design: This study was a prospective randomized controlled clinical trial.

Settings: The research took place in the interventional pain unit of a tertiary center in the university hospital.

Methods: Fifty-eight patients complaining of cancer-related chronic pelvic and perineal pain were randomized to either the PRF + SHP group $(n=29)$, which received SHP-N combined with PRF of the sacral roots S2-4, or the SHP group $(n=29)$, which received SHP-N alone. The outcome variables were the percentage of patients who showed a $>50 \%$ reduction in their Visual Analog Scale (VAS) pain score, the VAS pain score, and global perceived effect evaluated during a 3-month follow-up period.

Results: The percentage of patients who showed a $>50 \%$ reduction in their VAS pain score was significantly higher in the SHP + PRF group compared to the SHP group when assessed at one month $(92.9 \%[\mathrm{n}=26]$ vs $57.7 \%[\mathrm{n}=15] ; P=.003)$ and 3 months $(85.7 \%[\mathrm{n}=24)$ vs $53.8 \%[\mathrm{n}=14] ; P=$ .01) post procedure, respectively. However, no significant difference was observed between the 2 groups at the 6 -month evaluation (SHP + PRF [57.1\% $(n=16)]$ vs SHP $[50 \%(n=13)] ; P=.59)$. There was a statistically significant reduction of VAS in the SHP + PRF group in comparison to the SHP group at one month $(2.8 \pm 0.9$ vs $3.5 \pm 1.2$ [mean difference, -0.7 (95\% confidence interval $[\mathrm{Cl}$ ], -1.29 to -0.1$), P$ $=.01$ ]), 2 months $(2.8 \pm 0.9$ vs $3.5 \pm 1.2$ [mean difference, $-0.64(95 \% \mathrm{Cl},-1.23$ to -0.05$), P=.03$ ]), and 3 months $(2.7 \pm 1$ vs $3.4 \pm 1.2$ [mean difference, $-0.67(95 \% \mathrm{Cl},-1.29$ to -0.05$)$ ], $P=.03$ ]) post procedure, respectively; however, the 2 groups did not significantly differ at 2 weeks, 4, 5, and 6 months post procedure. Regarding postprocedural analgesic consumption, there were trends towards reduced opioid consumption at all postprocedural measured time points in the SHP+PRF group compared to the SHP group; these differences reached statistical significance at 2 months (median, 30 [interquartile range (IQR), 0.00-30] vs median, 45 [IQR, 30-90]; $P=.046$ ) and 3 months (median, 0.00 [IQR, 0.00-30] vs median, 30 [IQR, 0.00-67.5]; $P=.016$ ) post procedure, respectively. Limitations: The study follow-up period is limited to 6 months only.

Conclusions: SHP-N combined with PRF of the sacral roots $(\mathrm{S} 2,3,4)$ provided a better analgesic effect than SHP-N alone for patients with chronic pelvic and perineal pain related to pelvic cancer.

Trial Registry: ClinicalTrials.gov. NCT03228316.

Key words: Pelvic pain, pulsed radiofrequency, sacral roots, superior hypogastric plexus

Pain Physician 2020: 23:149-157 
hronic pelvic cancer pain is a disabling condition emanating from tumor involvement Jof the pelvic viscera, pelvic muscular and neural structures (1). Various studies reveal that the prevalence of pain in patients with pelvic malignancy is $>50 \%$ and can rise up to $60 \%$ to $70 \%$ in patients with advanced or metastatic disease $(2,3)$.

Analgesic drugs, specifically opioids, are the mainstay of cancer pain management. However, interventional denervation therapy is needed in $10 \%$ to $15 \%$ of patients with intractable cancer pain resistant to conventional analgesics (4).

The pelvis is heavily innervated by a complex network of sympathetic, parasympathetic, and somatic nerve fibers. The sympathetic outflow to the pelvic viscera arises from the thoracolumbar spinal cord segments (T12-L2) and is conveyed through the superior hypogastric plexus (SHP), which resembles the pelvic extension of aortic sympathetic plexuses and contains afferent pain fibers from most of the pelvic structures: the bladder, urethra, uterus, perineum, prostate, rectum, and descending colon $(5,6)$. The parasympathetic outflow is carried on sacral roots (S2-S4) that converge into the preganglionic pelvic splanchnic nerves and join the hypogastric nerves "condensation of sympathetic fibers descending from SHP" (5) to form the inferior hypogastric plexus around the distal part of the rectum, lower part of the bladder, prostate, seminal vesicles, uterine cervix, and vaginal fornices. Lastly, the somatic efferent and afferent innervation to the pelvis originates from sacral spinal cord levels S2-S4.

Chronic pelvic pain due to cancer is complex and usually involves an interplay of neuropathic, visceral, and somatic mechanisms.

Superior hypogastric plexus neurolytic (SHP-N) block is the mainstay management for pelvic cancer pain of visceral origin when oral opioids fail due to inefficacy or intolerance to side effects. Unfortunately, SHP-N has the potential to control pelvic pain in $62 \%$ to $72 \%$ of patients at best $(5-8)$ because chronic pelvic pain may assume additional characteristics other than visceral. This might be due to alternate pathways for pain conduction via the sacral pathway.

Sacral neuromodulation has been used to control a variety of forms of pelvic pain $(9,10)$. Siegel and colleagues (9) conducted a feasibility study in patients with intractable pelvic pain. They showed that sacral nerve stimulation decreased the severity and duration of the pain with improvement in quality of life.

Since sacral roots have been a target for neuro- modulation in chronic pelvic pain syndromes (11), specifically interstitial cystitis (12), prostadynia (13), vulvodynia (14), and coccydynia (15), the authors hypothesize that sacral roots could be a potential target for pulsed radiofrequency (PRF), which is widely used for a diversity of chronic pain states (16). Specifically, combining SHP-N with PRF of the sacral roots might block most of the pain emanating from the pelvic structures and improve the success rate of SHP-N in controlling pelvic and perineal cancer pain.

\section{Methods}

After obtaining the ethical committee approval of our institutional review board and signed informed written consent from each patient, which included explanation of the procedure, the benefits, the risks, and the alternatives, 58 patients suffering from chronic pelvic and perineal cancer pain were enrolled. The study is registered at ClinicalTrials.gov with unique ID: NCT03228316.

The inclusion criteria were adult patients with chronic pelvic and perineal pain due to pelvic cancer or postsurgical pain after surgery for pelvic cancer; and a Visual Analog Scale (VAS) pain score $\geq 5$ on a 0-10 scale despite treatment with a standard analgesic regimen that included oral opioids, acetaminophen, and amitriptyline, or intolerance to these analgesics.

The exclusion criteria were coagulopathy, infection at the site of injection, severe cardiac compromise intolerant to sympathetic block, and patients who could not tolerate the prone position.

Patients were randomly assigned to 2 equal groups, using a computer-generated list of numbers that were masked in opaque sealed envelopes and opened before the procedure. The SHP + PRF group $(n=29)$ received SHP-N combined with PRF of the sacral roots S2-S4; the SHP group $(n=29)$ received SHP-N alone.

\section{SHP-N Procedure}

The SHP-N procedure (17) went as follows: The patient was placed prone on the radiolucent table with a pillow under the pelvis to correct lumbar lordosis. An intravenous line was established and one liter of normal saline was administered. Basic monitors (pulse oximeter, electrocardiogram, and noninvasive blood pressure) were connected to the patient and $5 \mathrm{mg}$ of midazolam was given for sedation. Firstly, an anteroposterior (AP) fluoroscopic image was obtained with cephalic orientation to align the lower border of L5. Next, the C-arm was oriented to $20^{\circ}-25^{\circ}$ obliquely, and 
the point of entry was marked at the level of the L5-S1 disc (in the bone free widow). Three $\mathrm{mL}$ of $1 \%$ lidocaine was infiltrated, and a $15-\mathrm{cm}, 20$-gauge curved tip needle was inserted in the direction of the beam (end-on). The needle was advanced under the oblique view, hugging the lateral margin of the L5 vertebra or L5-S1 disc until a reasonable depth was obtained $(7-8 \mathrm{~cm})$. Under the lateral view, the needle was further inserted until it reached the anterolateral margin of the vertebral body; 1 to $2 \mathrm{~mL}$ of contrast medium (iohexol, Omnipaque; GE Healthcare Inc., Ireland, Cork, Ireland, $300 \mathrm{mg} \mathrm{I} / \mathrm{mL}$ ) was injected after negative aspiration to outline smoothly the L5-S1 junction (smooth contour of the contrast medium is due to anterior psoas fascia), both up and down without posterior spread toward the nerve root, "C-shape with the concavity faces posteriorly" (17) (Fig. 1). The AP view was obtained to ensure favorable dye position, "paramedian globular at the L5-S1 position" (17) (Fig. 2). Next, $6 \mathrm{~mL}$ of a mixture of lidocaine $2 \%$ and alcohol $100 \%$ (1:1) was injected, and $1 \mathrm{~mL}$ of lidocaine was injected during needle withdrawal. The same procedure was repeated on the other side.

\section{Radiofrequency of the Sacral Roots Procedure}

An AP image with cephalic orientation and slight obliquity was obtained to visualize the sacral foramina (S2-S4) clearly as circuits or hemi-circuits; in this position, the anterior sacral foramina are on the same trajectory with the posterior ones. The needle entry point was in the upper outer margin of the selected foramen. A 10$\mathrm{cm}$ curved tip radiofrequency (RF) cannula with a $1-\mathrm{cm}$ active tip needle was introduced (Fig. 3); then a lateral view was obtained to visualize the needle tip residing just anterior to the sacrum. At this point, the threshold of sensory stimulation was below $0.6 \mathrm{~V}$ in all cases. For the $\mathrm{S} 2$ root, the patient felt pain in the lower limb and pelvis; for the S3 root, the patient felt pain deep in the pelvis and external genital organs; and for the S4 root, the patient felt perianal pain. After the 3 needles were in place at the desired target (Fig. 4), 5 cycles of PRF, 2 minutes each, were delivered. PRF was applied in 20-millisecond pulses every 500 milliseconds (20 ms of $500-\mathrm{kHz}$ RF pulses, delivered at a rate of $2 \mathrm{~Hz}$ ). Maximum temperature voltage was automatically controlled to $42^{\circ} \mathrm{C}$; then the needles were removed and the same procedure was repeated on the other side.

The PRF procedures were performed by the same investigator (senior staff pain clinician) and all followups were carried out by another investigator who was not aware of the type of intervention performed.

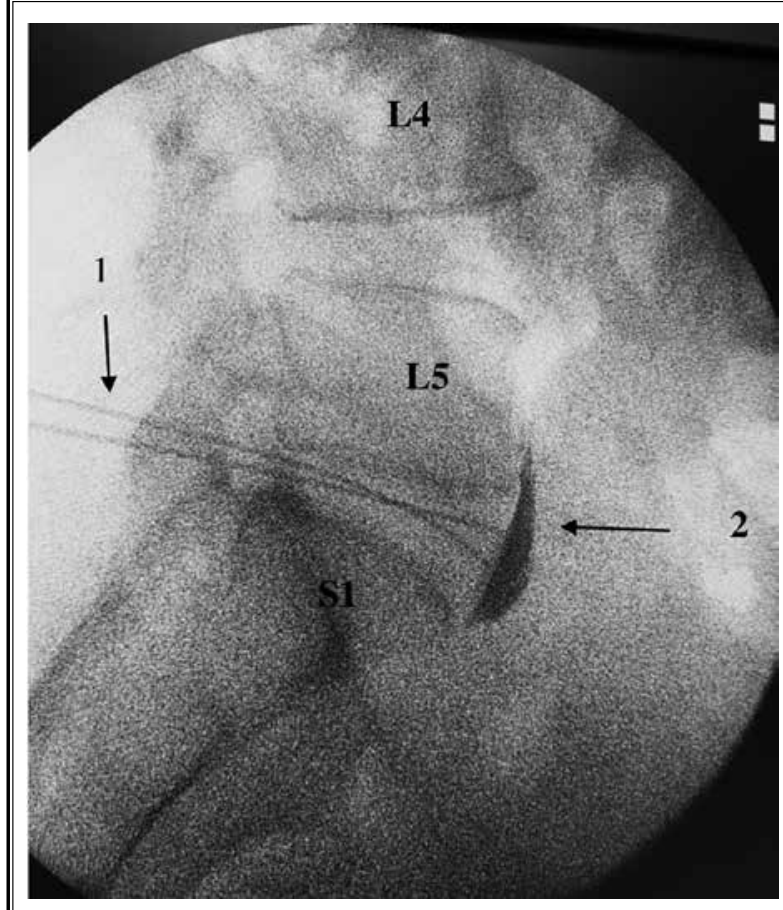

Fig. 1. A lateral x-ray image of the lumbosacral region, illustrating the final needle tip position anterior to the L5S1 disc.

$\mathrm{L} 4=$ the fourth lumbar vertebra. $\mathrm{L} 5=$ the fifth lumbar vertebra. $\mathrm{S} 1=$ the first sacral vertebra. $1=$ the 2 needles pass paravertebrally to reach the final target (anterior to the L5-S1 disc). $2=$ the characteristic dye distribution of SHP-N (C-shaped with the concavity facing posteriorly and smooth outlines).

The primary outcome variable was the percentage of patients who showed $>50 \%$ reduction in their VAS pain score (from baseline values), as measured at 1, 3, and 6 months post procedure. The secondary outcome variables were the changes in level of pain intensity, as measured by a VAS pain score; and daily morphine equivalent-consumption at 2 weeks and 1, 2, 3, 4, and 6 months following the procedure. The conversion to morphine was approximately as follows: tramadol to morphine $=10: 1$; hydromorphone to morphine $=1: 5$; transdermal fentanyl to morphine $=25 \mathrm{mcg} / \mathrm{h}$ equals $60 \mathrm{mg}$ morphine; $50 \mathrm{mcg} / \mathrm{h}$ equals $120 \mathrm{mg}$ morphine; and $100 \mathrm{mcg} / \mathrm{h}$ equals $240 \mathrm{mg}$ morphine. Also, the global perceived effect (GPE) was assessed at 3 months following the procedure. The GPE was assessed by a 7-point Likert-like verbal rating scale where: extremely dissatisfied $=1$, dissatisfied $=2$, somewhat dissatisfied $=3$, undecided $=4$, somewhat satisfied $=5$, satisfied $=$ 6 , and extremely satisfied $=7$. 


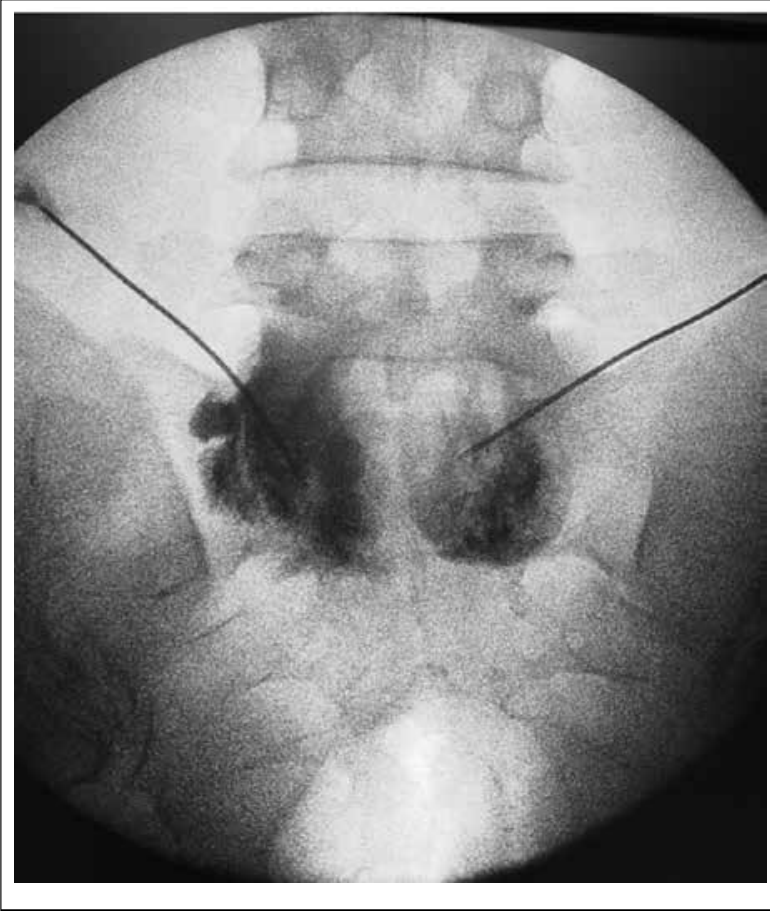

Fig. 2. A posteroanterior $x$-ray image of the lumbosacral region, illustrating the final needle tip position at L5-SI bilaterally and the characteristic distribution of dye of SHP-N (globular).

\section{Statistical Analysis}

The statistical analysis was carried out on a personal computer using SPSS Version 22.0 (IBM Corporation, Armonk, NY). The normality of continuous data distribution was tested with the Anderson-Darling test prior to further statistical analysis. Categorical data were described as number and percent, and comparisons were made by chi-square and Fisher exact tests. Continuous data were described as mean \pm standard deviation (SD) or $95 \%$ confidence interval $(\mathrm{Cl})$, and point-by-point comparisons were done by unpaired Student $t$ tests. A general linear model for repeated measures was used for analysis of VAS pain scores over time ( 2 weeks, 1 month, 2 months, 3 months, 4 months, 5 months, and 6 months post procedure), examining the following effects: group, time, and group-by-time interaction, followed by post-hoc tests with Bonferroni corrections for multiple comparisons. Medians and interquartile ranges were used for skewed data (GPE and morphine consumption), and comparisons were made using the Mann-Whitney test. $P<.05$ was considered statistically significant.

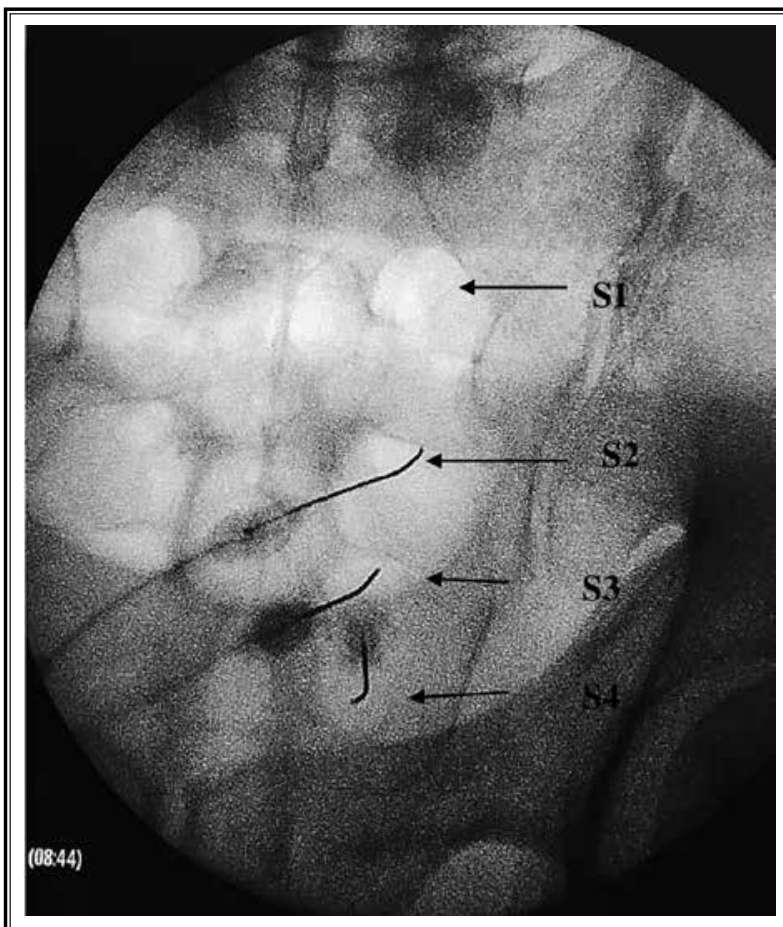

Fig. 3. A posteroanterior $x$-ray image of the lumbosacral region, illustrating the sacral foramina and the radiofrequency needles inside them.

$\mathrm{S} 1=$ the first sacral foramen. $\mathrm{S} 2=$ the second sacral foramen. $\mathrm{S} 3$ $=$ the third sacral foramen. $S 4=$ the fourth sacral foramen.

Based on a previous study (9), which reported that $62 \%$ of patients complaining of pelvic-related cancer pain managed with SHP-N showed more than $50 \%$ reduction in VAS pain scores from baseline values, we believed that PRF of the sacral roots $(S 2,3,4)$ combined with SHP-N would achieve more than $50 \%$ reduction in VAS pain scores from baseline values in $95 \%$ of patients. Using these assumptions, the estimated sample size for the 2-sample comparison of proportions using the Fisher exact test for 2 unequal independent groups revealed a minimum sample size of 26 patients in each study group assuming a study power of $80 \%$ and a type I error of .05. We enrolled 29 patients in each group to allow for drop-outs.

\section{Results}

Sixty patients were assessed for eligibility; 58 patients were allocated into 2 equal groups of 29 each. In the SHP + PRF group, one patient was excluded due to failure to target sacral roots and 28 patients remained for analysis. In the SHP group, 3 patients missed follow- 
up, and 26 patients remained for analysis (Fig. 5).

There was not a statistically significant difference between the 2 groups with respect to demographic data and patient characteristics (Table 1).

The percentage of patients who showed $>50 \%$ reduction in their VAS pain score was significantly higher in the SHP + PRF group compared to the SHP group when assessed at 1 month $(92.9 \%[n=26]$ vs $57.7 \%[n=$ $15], P=.003)$ and 3 months $(85.7 \%[\mathrm{n}=24]$ vs. $53.8 \%$ [n $=14], P=.01$ ) post procedure, respectively. However, no significant difference was observed between the SHP + PRF group (57.1\% [n $=16])$ and the SHP group (50\% [n $=13]$ ) at the 6 -month evaluation $(P=.59)$ (Table 1).

Using the general linear model, an analysis of VAS pain scores over time at 2 weeks, 1, 2, 3, 4, 5, and 6 months following the procedure revealed no statistically significant overall group difference between the SHP + PRF group (mean, $3.80 \pm$ standard error [SE], 0.21) and the SHP group (mean, $4.23 \pm \mathrm{SE}, 0.22$ ); mean difference: $-0.42(95 \% \mathrm{Cl},-1.03-0.18 ; P=.165)$. However, there were significant time $(P<.001)$ and group-by-time $(P<.003)$ interaction effects when the tests of within-subject effects and within-subject contrasts were applied.

Further point-by-point comparisons of the means of VAS pain scores at 2 weeks, 1, 2, 3, 4, 5, and 6 months following the procedure using the independent samples $t$ test revealed a statistically significant reduction in VAS scores in the SHP + PRF group vs the SHP group at 1 month (mean, $2.8 \pm \mathrm{SD}, 0.9$ vs $3.5 \pm 1.2$; mean difference, $-0.7[95 \% \mathrm{Cl},-1.29$ to -0.1$], P=.01), 2$ months (mean, $2.8 \pm S D, 0.9$ vs mean, $3.5 \pm S D, 1.2$; mean difference, -0.64 [95\% Cl, -1.23 to -0.05$], P=.03$ ), and 3 months (mean, $2.7 \pm \mathrm{SD}, 1$ vs mean, $3.4 \pm \mathrm{SD}, 1.2$; mean difference, -0.67 [95\% $\mathrm{Cl},-1.29$ to -0.05$], P=.03$ ) post procedure, respectively. However, the 2 groups did not significantly differ at 2 weeks, 4, 5, and 6 months post procedure (Table 2).

Regarding postprocedural analgesic consumption, there were trends towards reduced morphine consumption at all postprocedural measured time points in the SHP + PRF group compared to the SHP group; these differences reached statistical significance at 2 months (median, 30 [IQR, 0.00-30] vs median, 45 [IQR, 30-90], $P=.046$ ) and 3 months (median, 0.00 [IQR, 0.00-30] vs median 30 [IQR, 0.00-67.5], $P=.016)$ post procedure, respectively (Table 3 ).

The level of satisfaction with the procedure (GPE) was greater in the SHP + PRF group (median, 6.5 [IQR, 5-7]) compared to the SHP group (median, 4.5 [IQR, 2.75-7]) $(P=.045)$.

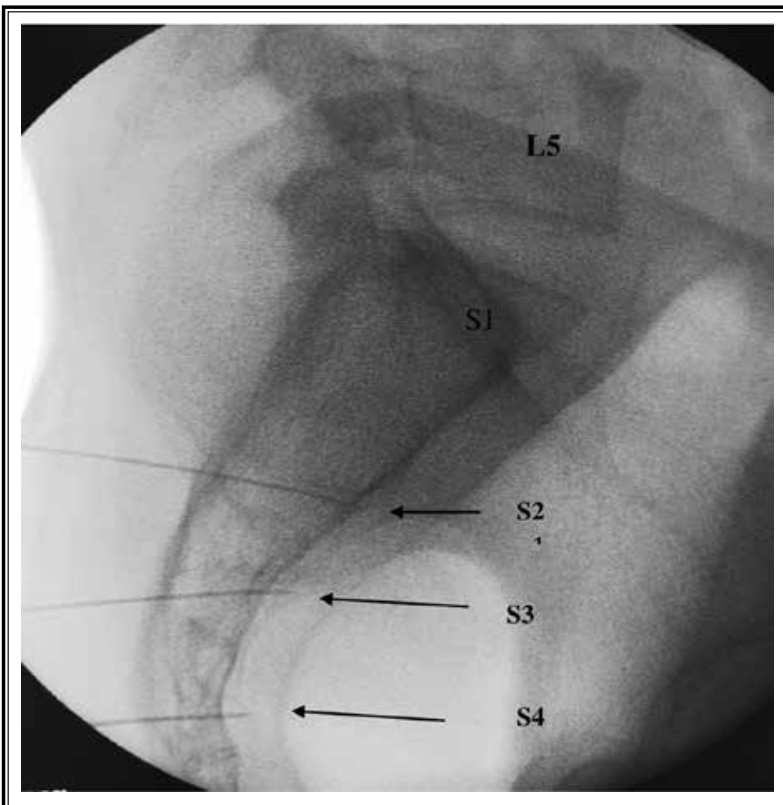

Fig. 4. A lateral $x$-ray image of the lumbosacral region, illustrating the radiofrequency needles passing through the sacral foramina $(S 2,3,4)$ and the needle tips situated just anterior to them.

$\mathrm{L} 5=$ the fifth lumbar vertebra. $\mathrm{S} 1=$ the first sacral vertebra. $\mathrm{S} 2=$ the needle pathway through the S2 foramen. S3 = the needle pathway through the $\mathrm{S} 3$ foramen. $\mathrm{S} 4=$ the needle pathway through the S4 foramen.

\section{Discussion}

SHP-N combined with PRF of the sacral roots (S2, 3, 4) provided a better analgesic effect than SHP-N alone for patients with chronic pelvic and perineal pain related to pelvic cancer.

It was obvious from our data analysis that a significantly greater number of patients in the SHP + PRF group continued to gain more than $50 \%$ reduction in their VAS pain scores until 3 months post procedure, compared to the SHP group (85.7\% [ $=24$ ] vs $53.8 \%$ [n $=14], P<.01$ ), respectively; consequently, lower VAS pain score values, less daily opioid consumption, and better satisfaction were achieved in the SHP + PRF group. However, the additional analgesic effect of sacral roots PRF was short-lasting and only $57.1 \%$ ( $n=16$ ) of patients continued to have more than $50 \%$ reduction in their VAS pain scores until 6 months post procedure, compared to $50 \%(n=13)$ of patients in the SHP group $(P<.59)$.

To our knowledge, this is the first study in the literature to use PRF of the sacral roots as an analgesic 


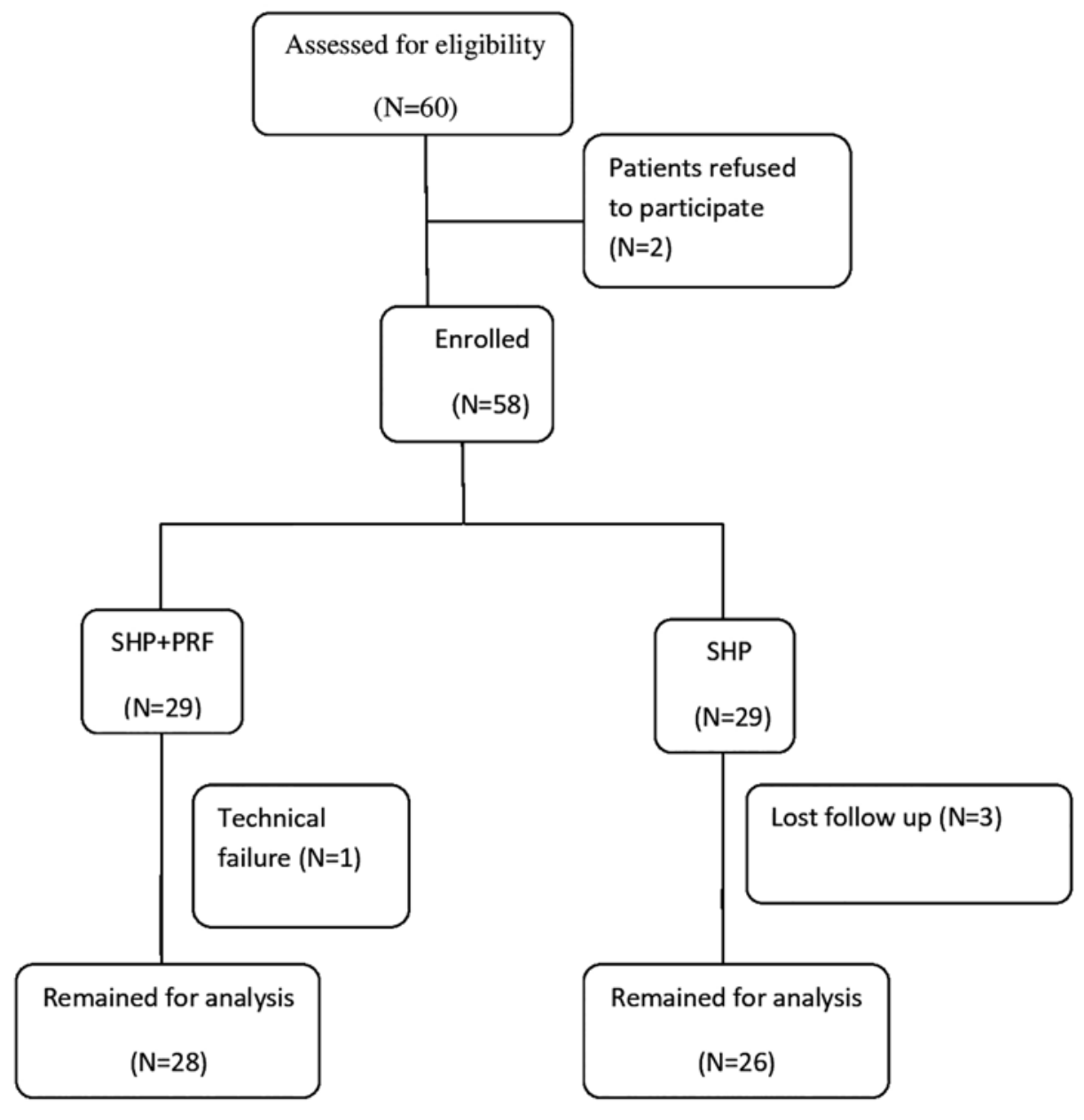

Fig. 5. The flowchart of the patients through the study.

modality to relieve pelvic and perineal cancer pain. This idea was inspired from the usage of sacral roots as a target for neuromodulation in many chronic pelvic pain syndromes.

The success rate of SHP-N reported in the literature ranges from $60 \%$ to $70 \%(5,7,8)$. In 1990 , Plancarte et al (7) reported a $70 \%$ reduction in pain VAS scores in 28 pelvic cancer patients. In 1993, de Leon-Casasola and colleagues (5) included 26 patients with intractable chronic pelvic pain due to advanced gynecologic, colorectal, or genitourinary cancer, and they found that 18 patients $(69 \%)$ had satisfactory pain relief, and 3 patients (12\%) required a second attempt of SHP-N for pain relief. In 1997, Plancarte et al (8) evaluated 227 patients with poorly controlled pelvic pain due to gyne- cologic, colorectal, or genitourinary malignancies and revealed that satisfactory pain relief had been achieved in $62 \%$ of patients after the first blockade and in $72 \%$ of patients after the second blockade.

The high failure rate of SHP-N, approximately $30 \%$ to $40 \%$, can be explained by several factors: firstly, SHP is a large network of nerve fibers distributed massively around pelvic organs, so a block of the plexus needs a large volume of neurolytics that cannot be injected due to nearby somatic nerves; secondly, SHP-N relieves pelvic pain of visceral origin, but is not effective against pain emanating from somatic structures.

Unfortunately, the previous studies $(18,19)$ that highlighted the success rate of SHP-N did not determine in their inclusion criteria the stage of pelvic cancer, 
PRF of the Sacral Roots for Pelvic and Perineal Cancer Pain

whether early or locally advanced "infiltrating the supporting muscles and ligaments or infiltrating the surrounding somatic nerves," (18) and also did not determine if the patients had had surgery for pelvic cancer, i.e., chronic postsurgical pain, which is mainly neuropathic. Moreover, these studies did not mention if the patient had been treated with radiotherapy, which is known to cause neuropathy as a side effect.

So, the improved success rate in the SHP + PRF group could be attributed to the blocking of pain signals that could partially pass through the sacral roots in addition to the main pathway, the superior hypogastric plexus; thus, PRF could block pain-firing through the sacral roots when there is extra-organ extension of the tumor or nerve injury due to surgical intervention for pelvic cancer.

Supporting our hypothesis is that sacral neuromodulation has been used successfully for treatment of pain arising from pelvic viscera. Siegel and colleagues (9) conducted a feasibility study in patients with intractable pelvic pain. They showed that sacral nerve stimulation decreased the severity and duration of the pain with improvement in quality of life. Similarly, Comiter (20) performed a prospective study on patients with interstitial cystitis treated with sacral neuromodulation and revealed that $94 \%$ demonstrated sustained pain improvement.

The duration of pain relief, 3 months, provided by
Table 1. Demographic data, patients' characteristics, and patients with $>50 \%$ postprocedural reduction in VAS pain score during 6 months of follow-up ( $n, \%)$.

\begin{tabular}{|l|c|c|c|}
\hline \multicolumn{1}{|c|}{ Variable } & $\begin{array}{c}\text { SHP + PRF } \\
(\mathbf{n = 2 8})\end{array}$ & $\begin{array}{c}\text { SHP } \\
(\mathbf{n = 2 6 )}\end{array}$ & $\boldsymbol{P}$ Value \\
\hline Age & $60 \pm 10$ & $59 \pm 11$ & .98 \\
\hline Gender (men/women) & $17 / 11$ & $18 / 8$ & .87 \\
\hline BMI & $24 \pm 6$ & $25 \pm 7$ & .97 \\
\hline Locally advanced cancer bladder & 6 & 6 & - \\
\hline Postradical hysterectomy & 4 & 4 & - \\
\hline Locally advanced colorectal cancer & 3 & 4 & - \\
\hline $\begin{array}{l}\text { Locally advanced anal cancer (abdomino-perineal } \\
\text { resection) }\end{array}$ & 2 & 1 & - \\
\hline Cancer colon, colorectal anastomosis & 7 & 8 & - \\
\hline Cancer prostate with pelvic deposits & 1 & 0 & - \\
\hline Locally advanced cancer cervix & 2 & 2 & - \\
\hline Rectovaginal fistula (postsurgical) cancer rectum & 1 & 0 & - \\
\hline Total pelvic exenteration for recurrent cancer rectum & 2 & 1 & - \\
\hline No. patients (\%) $>50 \%$ VAS reduction at 1 mo & $26(92.9 \%)$ & $15(57.7 \%)$ & .003 \\
\hline No. patients (\%) $>$ 50\% VAS reduction at 2 mos & $24(85.7 \%)$ & $14(53.8 \%)$ & .01 \\
\hline No. patients (\%) $>50 \%$ VAS reduction at 3 mos & $16(57.1 \%)$ & $13(50 \%)$ & .59 \\
\hline
\end{tabular}

Abbreviations: BMI, body mass index; PRF, pulsed radiofrequency; SHP, superior hypogastric plexus; VAS, Visual Analog Scale.

Data are presented as number (\%) and mean \pm standard deviation (SD).

PRF of the sacral roots is concordant with application of PRF to other areas of pain. Hetta and colleagues (21) applied PRF to the ilioinguinal nerve and the genital branch of the genitofemoral nerve for patients with chronic postgroin surgery orchialgia and found that the percentage of patients showing $>50 \%$ reduction in their VAS pain scores after 3 months post procedure was $80 \%$ (24 out of 30 ) in the PRF group versus $23.33 \%$ (7 out of 30 ) in the sham group. Moreover, Cohen et al (22) retrospectively evaluated interventions for a diversity of chronic postsurgical pain in the thoracic region; patients received either PRF on dorsal root ganglion (DRG), PRF on intercostal nerve (ICN), or medical therapy and found that, at 3-months follow-up, $53.8 \%$ of patients in the DRG group continued to report $\geq 50 \%$ pain relief vs $19.9 \%$ in the medical therapy group and $6.7 \%$ in the ICN group.

The analgesic effect of PRF is thought to be due to the effects of electromagnetic waves that induce neuroplastic changes rather than thermal destruction (23). Hagiwara and colleagues (24) claimed that PRF potentiates the noradrenergic and serotonergic descending pain inhibitory pathways and inhibits excitatory nociceptive C-fibers. Moreover, Vallejo et al (25) found that proinflammatory cytokines such as tumor necrosis factor- $\alpha$ and interleukin- 6 were reduced in neural tissues exposed to PRF. Recent studies have publicized that PRF upregulates c-fos expression in laminae I and II of the dorsal horn (26). We believe that the prolonged and greater analgesic effects of PRF of the sacral roots are due to suppression of the activation of microglia cells and the p38-signaling pathway that occurs after nerve injury; microglia transform to reactive phenotype and display a progressive series of cellular and molecular changes, including morphological hypertrophy, rapid proliferation, upregulated expression of various genes, 
Table 2. Postprocedural VAS pain score.

\begin{tabular}{|l|c|c|c|c||}
\hline \multicolumn{1}{|c|}{ Variable } & $\begin{array}{c}\text { SHP + PRF } \\
(\mathbf{n}=\mathbf{2 8})\end{array}$ & $\begin{array}{c}\text { SHP } \\
(\mathbf{n}=\mathbf{2 6})\end{array}$ & $\begin{array}{c}\text { Mean Difference } \\
(\mathbf{9 5 \%} \text { CI) }\end{array}$ & $\boldsymbol{P}$ Value \\
\hline VAS, basal & $7.4 \pm 1.1$ & $7.1 \pm 1.1$ & $0.27(-0.35-0.9)$ & .38 \\
\hline VAS, 2 wks & $4.9 \pm 1.3$ & $5.3 \pm 1.2$ & $-0.4(-1.1-0.28)$ & .23 \\
\hline VAS, 1 mo & $2.8 \pm 0.9$ & $3.5 \pm 1.2$ & $-0.7(-1.29$ to -0.1$)$ & .01 \\
\hline VAS, 2 mos & $2.8 \pm 0.9$ & $3.5 \pm 1.2$ & $-0.64(-1.23$ to -0.05$)$ & .03 \\
\hline VAS, 3 mos & $2.7 \pm 1$ & $3.4 \pm 1.2$ & $-0.67(-1.29$ to -0.05$)$ & .03 \\
\hline VAS, 4 mos & $3.1 \pm 1.5$ & $3.6 \pm 1.4$ & $-0.43(-1.24-0.37)$ & .28 \\
\hline VAS, 5 mos & $3.2 \pm 1.5$ & $3.6 \pm 1.5$ & $-0.40(-1.26-0.45)$ & .35 \\
\hline VAS, 6 mos & $3.1 \pm 1.5$ & $3.5 \pm 1.5$ & $-0.36(-1.18-0.46)$ & .38 \\
\hline
\end{tabular}

Abbreviations: PRF, pulsed radiofrequency; SHP, superior hypogastric plexus; VAS, Visual Analog Scale.

Data are presented as mean $\pm \mathrm{SD}$, mean difference $(95 \% \mathrm{CI})$.

Table 3. Postprocedural MST requirements during 6 months of follow-up.

\begin{tabular}{|l|c|c|c|}
\hline \multicolumn{1}{|c|}{ Variable } & $\begin{array}{c}\text { SHP + PRF } \\
(\mathbf{n}=\mathbf{2 8})\end{array}$ & $\begin{array}{c}\text { SHP } \\
(\mathbf{n}=\mathbf{2 6})\end{array}$ & P Value \\
\hline MST, basal & $60(60-120)$ & $90(60: 120)$ & .622 \\
\hline MST, 2 wks & $70(60-120)$ & $90(60-120)$ & .761 \\
\hline MST, 1 mos & $60(30-60)$ & $60(30-90)$ & .276 \\
\hline MST, 2 mos & $30(0.00-30)$ & $45(30-90)$ & .046 \\
\hline MST, 3 mos & $0.00(0.00-30)$ & $30(0.00-67.5)$ & .016 \\
\hline MST, 4 mos & $0.00(0.00-60)$ & $45(0.00-90)$ & .115 \\
\hline MST, 5 mos & $0.00(0.00-90)$ & $45(0.00-90)$ & .290 \\
\hline MST, 6 mos & $0.00(0.00-90)$ & $60(0.00-100)$ & .366 \\
\hline
\end{tabular}

Abbreviations: MST, morphine sustained release tablet; PRF, pulsed radiofrequency; SHP, superior hypogastric plexus; VAS, Visual Analog Scale.

Data are presented as median (interquartile range [IQR]).

increased expression of microglia characteristic markers such as Iba1, and increased p38 phosphorylation in the spinal microglia cells (27).

\section{Technical Aspects}

For PRF of the sacral roots, it was not an easy job to navigate through the posterior sacral foramina to land in front of anterior ones, due to anatomical variability in the configuration of the sacrum. In the current study, we had 4 cases in which we were unable to go anteriorly to one or more of the anterior sacral foramina; instead, we went through the posterior foramen and landed just posterior to the anterior sacral plate.

For SHP-N, we had 5 cases in which the needle tip was inadvertently placed inside the iliac vessels; therefore, we changed the approach in these cases to a posteromedian transdiscal approach rather than the extradiscal one. Also, we preferred to use a curved tip needle instead of the straight chiba needle that was commonly used in previous studies, as the curved tip allows for navigation away from the L5 root which is frequently encountered in the needle pathway when an extradiscal paravertebral approach is used for SHP-N.

One study limitation that deserves mentioning is that we could not fix a certain opioid protocol for all cases; instead we relied on opioid conversion to morphine sustained release tablet (MST).

For future studies, a caudal catheter with an active tip capable of delivering PRF may help make the procedure more successful and quicker. Also, the procedure can be repeated at 3 months, which may be an economical way to treat patients at the end of life.

\section{Conclusion}

In conclusion, SHP-N combined with PRF of the sacral roots $(S 2,3,4)$ provided a better analgesic effect than SHP-N alone for patients with chronic pelvic and perineal pain related to pelvic cancer. 


\section{References}

1. Plancarte $R$, de Leon-Casasola $O A$ El- Helaly M, Allende S, Lema MJ. Neurolytic superior hypogastric plexus block for chronic pelvic pain associated with cancer. Reg Anesth 1997; 22:562-568.

2. Van den Beuken-Van Everdingen $\mathrm{MH}$, Rijke JM, Kessels AG, Schouten HC, van Kleef $M$, Patijn J. High prevalence of pain in patients with cancer in a large population-based study in the Netherlands. Pain 2007; 132:312-320.

3. Breivik H, Cherny N, Collett B, et al. Cancer-related pain: A pan-European survey of prevalence, treatment, and patient attitudes. Ann Oncol 2009; 20:1420-1433.

4. Cleeland CS, Gonin R, Hatfield AK, et al. Pain and its treatment in outpatients with metastatic cancer. $N$ Engl J Med 1994; 330:592-956.

5. De Leon-Casasola OA, Kent E, Lema MJ. Neurolytic superior hypogastric plexus block for chronic pelvic pain associated with cancer. Pain 1993; 54:145-151.

6. Kroll CE, Schartz B, Gonzalez-Fernandez $M$, et al. Factors associated with outcome after superior hypogastric plexus neurolysis in cancer patients. Clin J Pain 2014; 30:55-62.

7. Plancarte R, Amescua C, Patt RB, Aldrete JA. Superior hypogastric plexus block for pelvic cancer pain. Anesthesiology 1990; 73:236-329.

8. Plancarte SR, Guajardo RJ, Guillen NR. Superior hypogastric plexus block and ganglion impar. Tech Reg Anesth Pain Manag 2005; 9:86-90.

9. Siegel S, Paszkiewicz E, Kirkpatrick C, Hinkel B, Oleson K. Sacral nerve stimulation in patients with chronic intractable pelvic pain. J Urol 2001; 166:1742-1745

10. Mayer RD, Howard FM. Sacral nerve stimulation: Neuromodulation for voiding dysfunction and pain.
Neurotherapeutics 2008; 5:107-113.

11. Feler CA, Whitworth LA, Fernandez J. Sacral modulation for chronic pain conditions. Anesthesiol Clin North Am 2003; 21:785-795.

12. Whitemore KE, Payne CK, Diokno AC, Lukban JC. Sacral neuromodulation in patients with interstitial cystitis: A multicenter clinical trial. Int Urogynecol ] 2003; 14:305-309.

13. Anothaisintawee T, Attia J, Nickel JC, et al. Management of chronic prostatis/ chronic pelvic pain syndrome: A systematic review and network metaanalysis. JAMA 2011; 305:78-86.

14. Masheb RM, Nash JM, Brondolo E. Vulvodynia: Introduction and critical review of a chronic pain condition. Pain 2000; 86:3-10.

15. Mitra R, Cheung L, Perry P. Efficacy of fluoroscopically guided steroid injections in the management of coccydynia. Pain Physician 2007; 10:775-778.

16. Shi Y, Wu W. Treatment of neuropathic pain using pulsed radiofrequency: A meta-analysis. Pain Physician 2016; 19:429-444.

17. Prithvi Raj P, Erdine S. Pain-relieving procedures: The illustrated guide. In: Twycross A, Dowden S, Stinson J (eds). Interventional Pain Procedures in the Pelvic and Sacral Regions. 1st ed. Hoboken, NJ, John Wiley \& Sons Ltd, 2012: pp. 365-371.

18. Orlandini G. Selection of patients undergoing neurolytic superior hypogastric plexus block. Pain 1994; 56:121-122.

19. Bosscher $\mathrm{H}$. Blockade of the superior hypogastric plexus block for visceral pelvic pain. Pain Pract 2001; 2:162-170.

20. Comiter CV. Sacral neuromodulation for the symptomatic treatment of refractory interstitial cystitis: A prospective study. J Urol 2003; 169:1369-1373.
21. Hetta DF, Mahran AM, Kamal EE. Pulsed radiofrequency treatment for chronic post-surgical orchialgia: A double-blind, sham-controlled, randomized trial: Three-month results. Pain Physician 2018; 21:199-205.

22. Cohen SP, Sireci $A, W u$ CL, Larkin TM, Williams KA, Hurley RW. Pulsed radiofrequency of the dorsal root ganglia is superior to pharmacotherapy or pulsed radiofrequency of the intercostal nerves in the treatment of chronic postsurgical thoracic pain. Pain Physician 2006; 9:227-235.

23. Erdine S, Bilir A, Cosman ER, Cosman ER Jr. Ultrastructural changes in axons following exposure to pulsed radiofrequency fields. Pain Pract 2009; 9:407-417.

24. Hagiwara S, Iwasaka H, Takeshima N, Noguchi T. Mechanisms of analgesic action of pulsed radiofrequency on adjuvant-induced pain in the rat: Roles of descending adrenergic and serotonergic systems. Eur J Pain 2009; 13:249-252.

25. Vallejo R, Tilley DM, Williams J, Labak $S$, Aliaga L, Benyamin RM. Pulsed radiofrequency modulates pain regulatory gene expression along the nociceptive pathway. Pain Physician 2013; 16:E601-E613.

26. Haguichi $Y$, Nashold BS, Sluijter $M$, Cosman E, Pearlstein RD. Exposure of the dorsal root ganglion in rats to pulsed radiofrequency currents activities dorsal horn lamina I and II neurons. Neurosurgery 2002; 50:850-855

27. Liu R, Xu X, Xu Y, Fang X, Lin X. Pulsed radiofrequency on dorsal root ganglion relieved neuropathic pain associated with downregulation of the spinal interferon regulatory factor 8 , microglia, p38MAPK expression in a $\mathrm{CCl}$ rat model. Pain Physician 2018; 21:E307-E322. 
